\title{
PERFORMANCE, ATLETISMO, VO2MAX Y TIEMPO DE RESISTENCIA EN LA CINTA RODANTE DE ATLETAS DEL SEXO MASCULINO.
}

\author{
Lisímaco Vallejo Cuellar \\ Profesor \\ Departamento de Educación Física \\ Universidad Pedagógica Nacional \\ Maria Augusta Pedutti Dal Molin Kiss \\ Profesora \\ Escuela de Educación Física \\ Universidad de Sao Paulo.
}

\section{Resumen}

El seguimiento de la evolución del entrenamiento en el atletismo, ha sido realizado principalmente a partir de la evolución del consumo máximo de oxígeno; con todo, este criterio se ha mostrado insuficiente para un análisis más objetivo de la evolución, mantenimiento y disminución de la performance. Observamos que el con sumo de oxígeno se estabiliza y la performance continua mejorando. Se han analizado deportistas de nivel departamental y nacional, en diferentes temporadas (precompetitiva, competitiva y de transición), y se han realizado tests ergometritos en la bicicleta, en la Cinta rodante con el test tiempo de resistencia en la cinta rodante (Vallejo, C.L. 1988: Kiss y col. 1987), el cual ha sido utilizado a mejores performances (tiempo) en competiciones diferentes velocidades, de acuerdo con las de carreras en pista. Fueron estudiados tres grupo del sexo masculino, grupo 1 con un $n=3$, eran lanzadores, fueron examinados a una velocidad de15.6 km/h; el grupo 2 era de fondistas $n=5$ y examinados a una velocidad de $18 \mathrm{~km} / \mathrm{h}$ el grupo $3, \mathrm{n}=5$ eran velocistas y se evaluaron a una velocidad de $21.6 \mathrm{~km} / \mathrm{h}$ y el grupo $4, \mathrm{n}=8$ a una velocidad de $16.8 \mathrm{~km} / \mathrm{h}$, el grupo $5 \mathrm{n}=5$, corredores de media distancia, fueron examinados a una velocidad de $20.4 \mathrm{~km} / \mathrm{h}$, el grupo $6, \mathrm{n}=5$ fue examinado a una velocidad de $21.6 \mathrm{~km} / \mathrm{h}$. Los resultados de la evolución del grupo 1 fue significativa, lo que no ocurrió con los demás, hubo aumento, del tiempo de resistencia en la cinta entre las medidas primera y tercera época en todos los subgrupos.

Palabras claves: perfomance, atletismo, VO2MAX, tiempo, resistencia, cinta rodante, sexo masculino.

Desde hace algún tiempo el concepto de consumo máximo de oxígeno (VO2max) es reconocido como uno de los posibles criterios para diferenciar las actividades atléticas como manifiesta Bouhay y colaboradores, 1984; por tanto este es un factor importante pero no suficiente ara analizar las performances óptimas en carreras de larga y media distancia (Kiss y col., 1988; Vallejo, C.L. 1988; Fohrenbach y col., 1987; Bouhay y col., 1984; Costill y col., 1973).

Estudios realizados con tests que analizan el umbral de lactato como los de Vallejo y col., 1991, Kiss y col., 1987 y Heck y col., 1985; o como los estudios realizados analizando los umbrales ventilatorios como los de McLellan y Skinner en 1985, test determinan una velocidad umbral o límite, a partir de la cual comienza un aumento veloz de la concentración de ácido láctico sanguíneo, o también a la desproporcionada eliminación de gas carbónico. 
Por otra parte se viene intentando dimensionar la mayor eficiencia que los atletas entrenados tienen en carreras de 15y $20 \mathrm{~km} / \mathrm{h}$ según los estudios de Svedenhag y Sjodin, 1985; como también los trabajos de resistencia en la bicicleta ergometrica de Moritami y col., 1981 o en la cinta rodante Hugson y col., 1984; los cuales procuraron determinar para cada individuo una constante aeróbica y otra anaeróbica.

Una de las preocupaciones de las investigaciones es la necesidad de tests que cuantifiquen la evolución de la performance de deportistas en sus diferentes temporadas o etapas de la periodización del entrenamiento deportivo. Entre los trabajos con tests de resistencia en la cinta rodante, con velocidades relativamente altas en diferentes modalidades deportivas tenemos los de Kiss y col., 1978; Vallejo Cuellar, 1988; Kiss y col., 1988 y Vallejo y col., 1991 entre otros.

El objetivo de este trabajo es el de determinar las variable que muestran la evolución de la performance en grupos de deportistas brasileros del estado de Sao Paulo, de alto rendimiento en atletismo.

\section{Metodología}

Fueron estudiados tres muestras de atletas del sexo masculino, practicantes de diferentes especialidades en atletismo; la primera fue constituida por 13 atletas del proyecto "Adopte un Atleta" en el estado de Sao Paulo los cuales fueron avaluados en el laboratorio de Cineantropometría de la Escuela de Educación Física de la Universidad de Sao Paulo. La segunda muestra fue constituida por 8 atletas del proyecto "Futuro" del estado de Sao Paulo y la Tercera mestra fue constituida por 10 atletas del equipo de atletismo de la "Electropaulo".

Se subdividió la primera muestra en tres grupos en función de su especialidad y la velocidad utilizada en la cinta rodante.

Se realizaron las mediciones antropométricas de rutina, seguidas de un test submáximo en una bicicleta ergométrica electro-magnética, utilizándose el nomograma de Astrand y Ryhming, 1954 para el cálculo del consumo máximo de oxígeno.

Los cálculos iniciales para la velocidad en la cinta rodante para el test de resistencia (TR) fueron basados en los tiempos de carrera de los atletas (performance) en sus respectivas medias de velocidad en pista o campo. Por ejemplo un atleta que hacía los $3000 \mathrm{~m}$ en $9 \mathrm{~min} 41 \mathrm{~s}$, osea con una velocidad media de $18.59 \mathrm{~km} / \mathrm{h}$ y el cual, fue evaluado a $18 \mathrm{~km} / \mathrm{h}$, para la comparación en la misma época el campeón mundial de la prueba tenía una velocidad media de $24.21 \mathrm{~km} / \mathrm{h}$.

Los tres grupos de la primera muestra lo constituían el grupo 1 con 3 atletas lanzadores y valuados a una velocidad de $15.6 \mathrm{~km} / \mathrm{h}$; el grupo 2 con un $\mathrm{n}=5$ atletas corredores de larga distancia y media distancia y evaluados a una velocidad de $18 \mathrm{~km} / \mathrm{h}$ y el grupo $3 \mathrm{n}=5$, compuesto por velocistas examinados a $21.6 \mathrm{~km} / \mathrm{h}$.

Los atletas del proyecto futuro constituían el grupo 4 y fueron evaluados a una velocidad de 16,8 km/h (Vallejo, C.L. 1988).

Los atletas del equipo de la "Electropaulo" constituían los grupos 5 y 6 , los cuales fueron evaluados a las velocidades de $20.4 \mathrm{~km} / \mathrm{h}$ y $21.6 \mathrm{~km} / \mathrm{h}$ respectivamente. 
Los grupos 1,2,3 fueron examinados en el inicio, en la mitad y, al final de a: o y los atletas del Proyecto Futuro se examinaron en marzo de 1985 y marzo de 1986; y los grupos 5 y 6 fueron evaluados en 1.988 en su etapa precompetitiva y su segunda medida fue realizada después de tres meses (Etapa competitiva).

El tiempo de resistencia en la cinta en cada una de estas velocidades fue medido para cada atleta en cada una de las etapas del entrenamiento, hasta el agotamiento. El concepto de agotamiento fue clínico, manifestado por palidez, cianosis y el inicio de incoordinación motora, el atleta desconocía el tiempo que estaba haciendo.

El análisis de varianza a un factor con o sin datos repetidos, y a un nivel de significancia de 5\%; para los grupos 4, 5 y 6 se utilizó el test t de student (Glass y Hopkins, 1984), para los datos correlacionados.

\section{Resultados}

Los valores del peso y la estatura de los 6 grupos en el inicio de cada trabajo, son presentados en la Tabla 1. Hubo un aumento significativo del peso en todos los grupos, sin embargo esto no ocurrió con la estatura, la cual presentó diferencia significativa en el grupo 4.

En la Tabla 1, se presentan los resultados de la media (x) y la desviación standard (s), de los diferentes grupos, 1,2,3,4,5,6 (No.) y su respectiva muestra ( $n$ ) de algunas variables morfológicas como la edad (años), la estatura $(\mathrm{cm})$, peso $(\mathrm{kg})$, de atletas del sexo masculino.

Tabla 1

\begin{tabular}{|l|l|l|l|l|l|l|l|}
\hline \multicolumn{2}{|l|}{ Grupos } & \multicolumn{2}{l|}{ Variables morfol } \\
\cline { 3 - 8 } \multicolumn{2}{|l|}{} & \multicolumn{2}{l|}{ Edad (años) } & \multicolumn{2}{l|}{ Estatura (cm.) } & Peso & (Kg) \\
\hline No. & $\mathrm{n}$ & $\mathrm{x}$ & $\mathrm{s}$ & $\mathrm{x}$ & $\mathrm{s}$ & $\mathrm{x}$ & $\mathrm{s}$ \\
\hline 1 & 3 & 16,7 & 0,58 & 180,30 & 5,62 & 76,76 & 10,47 \\
2 & 5 & 16,6 & 1,14 & 170,94 & 8,99 & 60,90 & 9,25 \\
3 & 5 & 16,8 & 0,41 & 180,20 & 10,30 & 67,70 & 8,89 \\
4 & 8 & 16,8 & 0,30 & 174,30 & 3,27 & 62,80 & 3,48 \\
5 & 5 & 22,5 & 3,30 & 178,50 & 3,00 & 73,00 & 4,50 \\
6 & 5 & 23,5 & 4,30 & 176,40 & 4,30 & 74,00 & 3,30 \\
\hline
\end{tabular}

En la Tabla 2, se presentan los valores del consumo máximo de oxigeno (VO2max, en $1 / \mathrm{mm}$ y $\mathrm{ml} / \mathrm{kg} / \mathrm{mm}$ ), la evolución de las medidas 1,2 y 3 , en los diferentes grupos $1,2,3,4,5$ y 6 , sus valores medios $(x)$ y desviaciones standard (s), éstos resultados se obtuvieron de un análisis de varianza para datos repetidos, a un factor no significativo.

Tabla 2.

\begin{tabular}{|l|l|l|l|l|l|l|l|}
\hline \multirow{2}{*}{ GRUPOS } & \multicolumn{4}{l}{ CONSUMO MAXIMO DE OXIGENO V2MAX } \\
\cline { 3 - 8 } & $1 / \mathrm{min}$ & 2 & 3 & 1 & 2 & 3 \\
\cline { 3 - 8 } & 1 & 2 & 30,82 & 3,92 & 39,7 & 48,8 & 52,7 \\
\hline \multirow{2}{*}{1} & $\bar{x}$ & 3,02 & $3,6,78$ & 7,5 & 10,8 & 12,5 \\
\hline & $s$ & 0,69 & 0,69 & 0,78 &
\end{tabular}




\begin{tabular}{|l|l|l|l|l|l|l|l|}
\hline & $\bar{x}$ & 3,35 & 3,56 & 3,56 & 54,4 & 58,0 & 55,0 \\
& $s$ & 0,26 & 0,45 & 0,59 & 11,6 & 8,5 & 4,6 \\
\hline \multirow{2}{*}{3} & $\bar{x}$ & 2,95 & 3,15 & 3,15 & 44,2 & 48,0 & 45,4 \\
& $s$ & 0,24 & 0,50 & 0,70 & 9,1 & 13,2 & 9,9 \\
\hline \multirow{2}{*}{4} & $\bar{x}$ & 3,72 & - & 3,71 & 59,9 & - & 57,9 \\
& $s$ & 0,81 & - & 1,06 & 12,7 & - & 15,1 \\
\hline \multirow{2}{*}{5} & $\bar{x}$ & 3,85 & - & 3,90 & 60,0 & - & 59,5 \\
& $s$ & 0,40 & - & 0,40 & 8,2 & - & 15,1 \\
\hline \multirow{2}{*}{6} & $\bar{x}$ & 3,75 & - & 3,76 & 59,7 & - & 60,5 \\
& $s$ & 0,70 & - & 0,80 & 10,3 & - & 12,1 \\
\hline
\end{tabular}

El consumo máximo de oxígeno (VO2max) no presentó evolución significativa en ningún grupo, Tabla 2.

Tabla 3. Se presentan los valores del tiempo de resistencia en la cinta rodante en segundos (s), la evolución de las medidas 1, 2 y 3 , en los diferentes grupos 1,2,3,4,5 y 6 , sus valores médios $(x)$ y desviaciones standard (s), éstos resultados se obtuvieron de un análisis de varianza para datos repetidos, a un factor, *: significativo, ns:no significativo, al nivel de $5 \%$.

Tabla 3

\begin{tabular}{|c|c|c|c|c|c|}
\hline \multirow{2}{*}{\multicolumn{2}{|c|}{ GRUPOS }} & \multicolumn{4}{|c|}{$\begin{array}{l}\text { TIEMPO DE RESISTENCIA EN LA } \\
\text { CINTA RODANTE (s) }\end{array}$} \\
\hline & & 1 & 2 & 3 & $\mathrm{~F}$ \\
\hline 1 & $\begin{array}{l}\bar{x} \\
s\end{array}$ & $\begin{array}{l}88,5 \\
16,3\end{array}$ & $\begin{array}{l}103,7 \\
11,7\end{array}$ & $\begin{array}{l}268,3 \\
102,6\end{array}$ & ns \\
\hline 2 & $\begin{array}{l}\bar{x} \\
s\end{array}$ & $\begin{array}{l}101,4 \\
13,2\end{array}$ & $\begin{array}{l}170,8 \\
106,3\end{array}$ & $\begin{array}{l}345,4 \\
246,9\end{array}$ & * \\
\hline 3 & $\begin{array}{l}\bar{x} \\
s\end{array}$ & $\begin{array}{l}56,8 \\
26,2\end{array}$ & $\begin{array}{l}48,8 \\
26,5\end{array}$ & $\begin{array}{l}117,8 \\
50,7\end{array}$ & * \\
\hline 4 & $\begin{array}{l}\bar{x} \\
s\end{array}$ & $\begin{array}{l}451,3 \\
332,9\end{array}$ & $\begin{array}{l}- \\
-\end{array}$ & $\begin{array}{l}1041,1 \\
692,5\end{array}$ & * \\
\hline 5 & $\begin{array}{l}\bar{x} \\
s\end{array}$ & $\begin{array}{l}522,0 \\
340,6\end{array}$ & $\begin{array}{l}- \\
-\end{array}$ & $\begin{array}{l}830,8 \\
250,7\end{array}$ & * \\
\hline 6 & $\begin{array}{l}\bar{x} \\
s\end{array}$ & $\begin{array}{l}532,0 \\
239,9\end{array}$ & - & $\begin{array}{l}760,0 \\
220,8\end{array}$ & * \\
\hline
\end{tabular}

En relación al tiempo de resistencia en la cinta rodante hubo un aumento significativo entre la primera y tercera medidas, en el grupo 2,3,4,5 y 6 ; en el grupo 3 hubo diferencia significativa también entre la segunda y tercera medidas, Tabla 3.

\section{Discusión}

Los atletas del proyecto "Adopte un Atleta", tuvieron un aumento de peso solamente en el grupo 1; ninguno de los grupos presentó incremento de la variable aeróbica (VO2max), en $\mathrm{ml} / \mathrm{kg} / \mathrm{min}$, estando en desacuerdo con los trabajos realizados en escolares por Daniels y col., 1978 y de Kobayashi y col., 1978. 
Es importante resaltar que el tiempo de resistencia en la cinta mejoró en el grupo 2 en la primera fase o etapa de entrenamiento, sin embargo, la diferencia no fue significativa; el grupo 3, que fue evaluado a una velocidad de $21.6 \mathrm{~km} / \mathrm{h}$, presentó una estabilización de los resultados entre el primero y segundo test y con un aumento significativo en el tercer test.

Los atletas del grupo 4, "Proyecto Futuro", al igual que los atletas de los grupos 5 y 6 , de la "Electropaulo", también presentaron un aumento significativo en el tiempo de resistencia en la cinta rodante, mas no en la capacidad aeróbica o VO2max.

El aumento del tiempo de resistencia en la cinta se debió sí no probablemente, no sólo a una mejor eficiencia (Svedenhag y Sjodin, 1985), también a una mayor fracción del VO2max utilizada sin la producción de ácido láctico (Forenbach y col., 1987), sumado esto a una mayor capacidad anaeróbica.

De esta forma se sugiere que el seguimiento de la evolución del entrenamiento en atletismo sea realizado también con el test tiempo de resistencia en la cinta y no en la bicicleta ergométrica, teniendo en cuenta, que los tests de tiempo de resistencia en la cinta en velocidades elevadas, y utilizando las variables de la frecuencia cardiaca, el VO2max y la medición de la concentración de ácido láctico sanguíneo permitirán cuantificar objetiva e individualmente los componentes aeróbico y anaeróbico.

\section{Bibliografía}

ASTRAND, P.O. Y RYHMING, I. A nomogram for calculation of aerobic capacity (physical fitness)from pulse rate during submaximal work. Journal of Applied Physiology, Washington, 7 (20): 2 18-221, 1954

ASTRAND, P.O. Aerobic work capacity in men and women with special reference to age. Acta Physiologica Scandinavica, 49: (suppl. 169) 1960.

--------- Ergomketri Konditionsprov. A.B. Cykelfabriken Monark, Varverg, 1964.

ASTRAND, P.O. Y RODAHL, K. Tratado de fisiología do exercício. 2.ed., Rio de Janeiro, Ed. Interamerica, 1980.617p.

COSTILL , D.L. Y COL. Determinants of marathon running success. Internationale Zeitschrift fur Angewandte Physiologie, 29:249-254, 1971.

COSTILL, D.L. Y COL., Fractional utilization of the aerobic capacity during distance running. Medicine and Science in Sports, 5:248-252, 1973.

DANIELS, J. y Colaboradores. Differents and changes in V02 max among young runners 10-18 years of age. Med. Sci. Sports, 10(3): 200-203, 1978.

DANIELS, J. Physiological characteristics of champion athletes. Research Quarterly, 45:342-348, 1974a. .

-------- Running with Jim Ryun: a five-years study. Physician and Sportsmedicine, 2:6367, 1974b.

EKBLOM Y COL., Effect of training on circulatory response to exercise. Journal of Applied Physiology, 24(4):518-528, 1968.

FAULKNER, I.A. Physiology of swimming and diving. In: FALLS, H.B. Exercise Physiology. New York, Academic Press, 1968. P. 415-443.

FOHRENBACH, R.; MADER, A.B. Y HOLLMANN, W. Determination of endurance capacity and prediction of exercice intensities for training and competition in marathon runners. int. J. Sports Med., Stuttgart, 8(1): 11-8, 1987. 
GLASS, GB. Y HOPKINS, K.D. Statistical methods in education and psychology. 2ed., New Jersey, Printice-Hall, Inc., 1984. 578p.

GLESSER M.A. y VOGEL, J.A. Endurance capacity and a prediction of exercise on the bicycle ergometer. In:

McLELLAN, J.M.B.; SKINNER, J.S. Submaximal endurance related to the ventilation thresholds. Can. J. Appl. Sports Sci., Toronto, 10(2): 81-7, 1985.

HEGG, R.V. Y LUONGO, J. Elementos de biometría humana. 2 ed., Sao Paulo, Livraria Nobel, 1975. 173p.

HECK, G. y Colaboradores. Justification of the $4 \mathrm{mmol} / \mathrm{l}$ lactato thersholds. $\mathrm{mt}$. J. Sports Med., Stuttgart, 6(2): 117-30, 1985.

HUGHSON, R.L. y Colaboradores. A high velocity treadmill running test to assess endurance running potencial. int. J. Sports Med., Stuttgard, (5): 23-5, 1984.

KISS, M.A.P.D.M. Efeito do treinamento sobre a condicao cardiovascular de atletas do sexo femenino, con 13 a 15 anos de idade. Sao Paulo, 1972. (Tese de Doutoramento Instituto de Ciencias Biomedicas da Universidade de Sao Paulo).

KISS, M.A.P.D.M. Introducao a ciencia do esporte. Sao Paulo, EEFUSP, 1978.

KISS, M.A.P.D.M. y Colaboradores. Maximal time of endurance et high velocity in treadmill and aerobic condition in judo. In: World Cong Sports Medicine, 21, Brasilia, 1978, p. 247.

KISS, M.A.P.D.M. Avaliacao em Educacao Física: Aspectos biologicos e educacionais. Sao Paulo, Manole, 1987.

KISS, M.A.P.D.M. y Colaboradores. Limiar de lactato no teste de endurance escalonado em criancas e adolescentes. In: Simposio Internacional de Medicina Desportiva, 2o., Rio de Janeiro, 1987, p.12.

KISS, M.A.P.D.M.; VALLEJO, C.L.: MACHIDA, J.; CARVALHO, P.R. y RODRIGUEZ, R.L. Tempo de resistencia en esteira: Atletismo. Rev. Paul. Educ. Fis., Sao Paulo, 2(2):4953 ,

maio, 1988.

KLISSOURAS, V. Y COL., Adaptation to maximal effort: genetics and age. Journal of Applied Physiology, 35:288-293, 1973.

KOHAYASHI, K. y Colaboradores. Aerobic power as related to body growth and training in japanese boys: a longitudinal study. J. Appl. Physiology, 44: 666-72, 1978.

LARSON, L.A. Fitness, health and work capacity. International comitee for standardization of physical fitness tests. New York, Mac Millan, 1974. 594p.

LIESSEN, H. Comunicación pessoal. Sao Paulo, Escola de Educacao Fisica da Universidade de Sao Paulo, Outuvro de 1985.

McLELLAN, T.M. 7 SKINNER, J.S. Submaximal endurance performance related to the ventilation thersholds. Can. J., Appl. Sport Sci., Toronto, 10(2): 81-7, 1985.

MATSUI, H. Y COL., Maximum oxygen intake and its relation to body weight of japanese adolescents. Medicine Science in Sports, 4:27-32, 1972.

MORITAMI, T. y Colaboradores. Critical power as a measure of physical work capacity and a anaerobic threshold. In:

HUGSON, R.L.; OROK, C.J. y STAUDT, L.E. A high treadmill running to assess endurance running potential. Int. J. Sport Med., 5(1): 23-5, 1984.

MURASE, Y. Y COL., Longitudinal study of aerobic power in superior junior athletes., Medicine and Science in Sports and Exercise, 13(3):180-184, 1981.

NAGLE, F.J. Y COL., Maximal 02 uptake of boys and girls age 14-17. European Journal of Applied Physiology, 36:75-80, 1977.

OLIVEIRA, P.R. Resistencia aerobica en adolescentes e sua relacao com o crescimento e desenvolvimento físico: estudo longitudinal. Sao Paulo, 1981. (Dissertacao de Mestrado- Esola de educacao Física da Universidade de Sao Paulo). 
POLLOCK, M.L. Quantification of endurance training programs. In: WILMORE. J. Exercise and Sports Reviews, New York, Academic, 1973. p. 155-158.

SALTIN, B. Y COL., Response to exercise after bed rest and after training. Circulation, 38:(suppl.7) 1968.

SHEPHARD, R.J. Endurance fitness. Toronto, University of Toronto, Press, Canada, 1969.

SIEGEL, S. Estatística nao paramétrica. Para as ciencias do comportamento. Sao Paulo, Mcgraw-Hill, 1975. 350p.

SVEDENHAG, J. y SJODIN, B. Physiological characteristics of elite male runners in and off season. Can. J. Sport. Sci., Toronto, 10(3): 127-33, 1985.

TANNER, J.M. Growth at adolescence. 2 ed., Oxford, Blackwell Scientific Publications, 1962. 326p.

VALLEJO CUELLA.R, L. Análise da Capacidade aeróbica em atletas adolescentes. Tese de Mestrado em Educacao Física. Sao Paulo. Brasil, 1988.

VALLEJO, C.L.; LOMBANA, R. y SANCHEZ, C.E. Urnbral de lactato en atletas universitarios. En publicacion. 1991.

VALLEJO, C.L. y Colaboradores. Cineantropometria morfofuncional de atletas adolescentes. Revista Paulista de Educación Física, Sao Paulo-Brasil, 2(2):27-32, mayo, 1988.

VALLEJO, C.L. y Colaboradores. Cineantropometria morfofuncional de atletas adolescentes. Adaptado para la Revista de la Facultad de Ciencia y Tecnología, Santa Fé de Bogotá, Colombia, 1:27-31, enero 1991.

VALLEJO, C.L. y Colaboradores. Tempo de resistencia em esteira e lactato sanguíneo em atletas adultos. XVI Simposio de Ciencias do Esporte, Esporte de Alto Nivel, ANAIS, 69 outubro, 1988. Sao Bernardo do Campo, Brasil.

WILEY, J.P. y RHODES, E.L. The relationship of individual anaerobic thersholds to total. lactic and oxygen debts after a set treadmill run. Can. J. Appl. Sport Sci. Toronto, 11(1): 37-41, 1986.

WOLANSKI, N. Genetic and ecological components in the development of endurance fitness. In: OSTYN, M.: BEUNEN, G.: SIMONS, J.(eds). Kinanthropomery II, Baltimore, University Press, 1980. p.88-103. 